\title{
Light Regulated Osa-miR169e Is Implicated during Priming under High Temperature Stress in Rice
}

\author{
Akhilesh Kumar Kushawaha\#, Ambreen Khan\#, Sudhir Kumar Sopory, Neeti Sanan-Mishra* \\ Plant RNAi Biology Group, International Centre for Genetic Engineering and Biotechnology, New Delhi, India \\ Email: akhilesh2203@gmail.com, ambreen1211@gmail.com, Sopory@icgeb.res.in, „neeti@icgeb.res.in
}

How to cite this paper: Kushawaha, A.K., Khan, A., Sopory, S.K. and Sanan-Mishra, N. (2019) Light Regulated Osa-miR169e Is Implicated during Priming under High Temperature Stress in Rice. American Journal of Plant Sciences, 10, 1662-1674. https://doi.org/10.4236/ajps.2019.109118

Received: August 23, 2019

Accepted: September 26, 2019

Published: September 29, 2019

Copyright $\odot 2019$ by author(s) and Scientific Research Publishing Inc. This work is licensed under the Creative Commons Attribution International License (CC BY 4.0).

http://creativecommons.org/licenses/by/4.0/

\begin{abstract}
Plants face biotic and abiotic stresses during their entire life cycle, which leads to the loss in crop productivity. It has been shown that a relatively shorter exposure to heat stress, called priming, results in better adaptation of plants under subsequent stresses, which plants may face. While rice plants in nature often encounter high temperature stress conditions, the strategies to cope with those are poorly understood. We identified the involvement of microRNA pathways in the adaptation to heat stress (HS) at the physiological and molecular levels. It was observed that osa-miR169 levels are altered after HS and in response to light conditions. Its expression was also regulated by heat priming during anthesis and effectively responds to the successive exposure to high temperature stress during grain filling in rice. Osa-miR169 targets nuclear factor Y (NF-Y). We propose that osa-miR169: NF-Y regulatory module may be important for HS memory induced during high temperature priming and thus may serve to integrate stress responses with light regulated development. The future study in this direction will be useful to understand how plants acclimatize to the changing environment and thus help in generating stress tolerant crops.
\end{abstract}

\section{Keywords}

Abiotic Stress, Heat Priming, Light, Acclimation, MicroRNA

\section{Introduction}

Changes in the climatic conditions because of global warming are imposing challenges on plants by affecting the distribution of biotic and abiotic factors [1]. These stresses impact many important physiological processes, which lead to the loss in ${ }^{\#}$ Equal contribution. 
crop productivity. Plants have the ability to regulate their genetic machinery and metabolism in an attempt to withstand these stresses. Of the various factors, light and temperature play important roles in controlling plant development from vegetative to reproductive stages.

Several studies have shown that light signaling in plants is mediated by distinct transcriptional factors binding to light-responsive elements found within the promoters of light-regulated genes [2]. Analogous modulation of growth and development in plants is also mediated by temperature signals [3], however, any increase from ambient is perceived as stress. High temperature stress (HS) causes protein denaturation, increased fluidity of membrane lipids and elevated lipid peroxidation [4] leading to lower photosynthetic rate, reduced seed germination and emergence [5] [6]. At reproductive stages, it causes anther indehiscence, spikelet sterility, fruit abscission and lower rice seed-sets [7]. However a relatively shorter exposure to stress, like HS, light or chemical treatment [8], called priming, results in better adaptation of plants under subsequent stresses. The process of priming involves the activation of specific transcription factors (TFs), like HSFs that induce the expression of specific set of proteins, like HSPs [9], which in turn assist in cellular homeostasis [10]. Recently, it was demonstrated that light primes detoxification of the thermally induced reactive oxygen species during heat stress in Arabidopsis [11].

The known stress tolerance mechanisms are mediated through a regulatory network involving TFs, feedback circuits, etc. Numerous studies also report that small RNAs, especially the microRNAs (miRs) regulate gene expression at the transcriptional or post-transcriptional levels. MiRs are involved in various cellular and molecular pathways associated with almost every aspect of plant growth and development [12]. Small RNA analysis in Arabidopsis following HS identified the deregulation of miR159, miR164, miR166, miR169, miR172, miR319 and miR399 families [13]. Short exposure to HS deregulates the expression of miR156, miR159, miR162, miR169 and miR529 [14]. In rice cultivar N-22 miR159, miR166 and miR169 were shown to be deregulated under HS [15]. Another study involving heat stressed wheat tissues demonstrated significant down-regulation of miR172 and up-regulation of miR159, miR166 and miR169 [16]. Members of miR159, miR164, miR169, miR172, miR319 and miR399 families were found to be differentially regulated during HS in switchgrass [17]. The expression of miRs is also influenced by light. In Arabidopsis, miR163 expression is induced by light [18], while in rice miR164, miR166, miR167, miR168, miR169, miR530 and miR2879 are light inducible [19].

The biological functions of miR169 have been characterized in Arabidopsis, Barrel medic, maize, rice, switch grasses, sugarcane and tomato. It was shown that overexpression of miR169 or RNAi mediated knock down of its target transcript, MtHAP2-1 TF, control nodule development in Medicago truncatula [20]. The expression of miR169 was down-regulated under phosphate, nitrogen and sulfur deficient conditions [21]. In Tomato, miR169 was differentially regulated by either phosphate nutrition or arbuscular mycorrhizal fungi (AMF coloniza- 
tion) or both, indicating its regulatory role in the complex signal transduction networks of phosphate nutrition and/or AMF derived signaling [22]. In sugarcane, the miR169 was found to be deregulated in response to abiotic stress and water pollution [23]. It was reported that miR169 differentially expressed in switch grass under both heat and drought stress conditions [17]. The miR169 regulates a family of CCAAT box-binding TFs known as Nuclear Factor Y (NF-Y) in plants [24] [25]. The members of this family are known to enhance tolerance to abiotic stresses such as salinity [26], drought [27] and cold [28]. Members of the NF-Y family play essential roles in the control of flowering [29], seed development [30], photosynthesis [31] and nodule development [20].

We had earlier reported that integration of light and temperature signaling pathways in plants influence the miR based regulatory networks to regulate plant growth and development [32]. In this study, we report that osa-miR169 levels are altered after HS and in response to light conditions. Its expression is also regulated by heat priming during anthesis and effectively responds to the subsequent exposure to HS during grain filling in rice. The involvement of osa-miR169 and NF-Y regulatory module in the adaptation to recurring HS memory and thus may serve to integrate stress responses with light regulated development. This study will be useful to understand how plants acclimatize to the changing environment and thus can help design better strategies for generating stress tolerant crops.

\section{Materials and Methods}

\subsection{Plant Growth Conditions}

Heat-susceptible rice cultivar Pusa Basmati 1 (PB1) was chosen for this study. Seeds were surface sterilized with $0.1 \% \mathrm{HgCl}_{2}$ and Teepol, rinsed with water and then soaked in water overnight. Seeds were grown on germination sheets for two weeks in growth room at ICGEB, New Delhi and then transferred to growth chamber at $28^{\circ} \mathrm{C} \pm 2{ }^{\circ} \mathrm{C}$ and $14 / 10 \mathrm{~h}$ photoperiod for further experiments. The leaf tissue samples were harvested from 15-days old seedlings, while flag leaf tissues were collected during anthesis from plants grown under control conditions in the greenhouse.

\subsection{High Temperature Treatments}

The high temperature treatments were performed at different stages of plant growth. The initial experiments involved exposing two weeks old rice seedlings to temperatures of $33^{\circ} \mathrm{C}, 38^{\circ} \mathrm{C}$ and $43^{\circ} \mathrm{C}$, keeping $28^{\circ} \mathrm{C}$ as control growth temperature. Leaf tissues were harvested after $24 \mathrm{~h}, 48 \mathrm{~h}$ and $72 \mathrm{~h}$ of stress treatment, respectively.

The next set of experiments was performed during anthesis stage. Rice plants were divided into two groups. One group was given direct heat stress (not primed) and other was heat primed. In the first group, plants were exposed to high temperature at $42^{\circ} \mathrm{C}$ for $90 \mathrm{~min}$ during the light period. One set of plants was 
stressed before anthesis $(\mathrm{NBH})$ and the other after anthesis (NAH). The second group was heat-primed at $38^{\circ} \mathrm{C}$ for 90 min before anthesis (PBH) and after anthesis (PAH) and after one day gap, the primed plants were exposed to high temperature at $42^{\circ} \mathrm{C}$ for 90 minutes. In each set, plants grown at $28^{\circ} \mathrm{C}$ served as control. Each experiment was performed in triplicate using three plants in each set.

\subsection{Light Treatments}

These experiments were performed on two weeks old rice seedlings grown at normal dark/light cycle of $10 / 14 \mathrm{~h}$ at $28^{\circ} \mathrm{C}$ in greenhouse. One set of plants was transferred to continuous darkness (CD) and the second set was transferred to continuous light (CL) at normal temperature. Samples were harvested after $24 \mathrm{~h}$ and $48 \mathrm{~h}$ of light or dark exposure and used for further experimentation. Samples were harvested in three different replicates and each experiment was repeated thrice.

\subsection{Isolation of Total RNA}

Total RNA was extracted from various rice tissues using guanidine isothiocyanate (GITC) based protocol as described previously [33]. Briefly, leaf tissue was homogenised in liquid nitrogen and GITC buffer was added along with phenol and chloroform. The mixture was allowed to thaw slowly and centrifuged at high speed ( 13,000 rpm) for 15 mins. The aqueous phase thus obtained was extracted twice with phenol: chlorom form solution and kept for precipitation with ethanol. The pellet was washed twice using $75 \%$ ethanol at $13,000 \mathrm{rpm}$ for 15 mins each and dried at room temperature. The dried pellet of RNA was dissolved in required amount of DEPC-treated water and stored at $-20^{\circ} \mathrm{C}$.

\subsection{Quantitative RT-PCR Expression Analysis}

The expression levels of mature miR169 and their respective target were analyzed by qRT-PCR with slight modification using gene specific primers. Total RNA was used to synthesize cDNA using miR-specific stem-loop primer with $\mathrm{Su}$ perscript reverse transcript III (Invitrogen). For target gene, nuclear factor $\mathrm{Y}$ (NF-Y: LOC_Os07g06470), the cDNA was synthesized from isolated RNA using high capacity cDNA kit (Applied Biosystem, USA) and qRT-PCR were performed using SYBR Green Master Mix (Applied Biosystems, USA) according to the manufacture's recommendations for at least three experiments replicates and deviations are shown as error bars for each dataset. The normalized expression values were calculated with respect to $18 \mathrm{~S}$ control and relative fold change in expression was plotted. Control (C) was non-stressed rice plants grown at $28^{\circ} \mathrm{C} \pm$ $2^{\circ} \mathrm{C}$ and $14 / 10 \mathrm{~h}$ photoperiod (the primer sequences are provided in Table A1). To check the significance of the observed gene expression difference, the normalized mean values of each experiment was analyzed using the one-way analysis of variance (ANOVA) using Microsoft Excel. The treatment means were compared 
with their respective controls at a significance level of $\mathrm{P}<0.05$.

\section{Results and Discussion}

In an earlier study, using NGS based computational analysis, we showed that 45\% osa-miRs were deregulated under high temperature stress (HS) [32]. Among them osa-miR169 was one of the largest families represented by 19 members that were regulated by cues from light and HS. miR169 is a highly conserved family and its expression levels are influenced by different abiotic stress conditions [17] [23] [34]. Our previous study indicated that some members of miR169 family that were heat up-regulated in PB1, were differentially down-regulated or absent in the tolerant rice variety N22 [32]. It has been clearly demonstrated that miR169 negatively regulates the transcript level of NF-YA subunit [35] and thus may potentially regulate many aspects of plant life including primary root elongation, flowering, gametogenesis, seed development, abscisic acid signaling, and responses to HS, drought and light [36] [37].

\subsection{Expression of Osa-miR169 and Its Respective Target NF-Y Is Influenced by Light Duration}

To understand the influence of light, $\mathrm{PB} 1$ seedlings were grown at $28^{\circ} \mathrm{C} \pm 2{ }^{\circ} \mathrm{C}$ and $14 / 10 \mathrm{~h}$ photoperiod for two weeks and then transferred to continuous darkness (CD) or continuous light (CL). The expression patterns of osa-miR169 were checked in leaves of these seedlings after $24 \mathrm{~h}$ and $48 \mathrm{~h}$ of change in photoperiod. The normalized expression values were calculated with respect to internal controls and fold change in expression in CL and CD was plotted. The results showed that the overall expression of osa-miR169 decreased under both CL and $\mathrm{CD}$ relative to the control; however, the decrease was greater under $\mathrm{CD}$ (Figure 1(a)). At $24 \mathrm{~h} \mathrm{CD} \mathrm{(CD1),} \mathrm{a} \mathrm{decrease} \mathrm{in} \mathrm{osa-miR169} \mathrm{expression} \mathrm{up} \mathrm{to}$ 8 -folds was observed and the levels increased at $48 \mathrm{~h}$ (CD2). While under CL for $24 \mathrm{~h}$ (CL1), osa-miR169 expression reduced by half ( $\sim 5$-folds) and at CL for $48 \mathrm{~h}$ (CL2), a slight increase was observed in the expression of osa-miR169.

The expression of its target, NF-Y was also lowered as compared to the controls, although an inverse correlation between miR169 and NF-Y relative levels could be captured for both $\mathrm{CD}$ and CL conditions separately (Figure 1(b)). Its expression levels in CD1 and CL1 were higher than their corresponding counterparts at $48 \mathrm{~h}$ time point. This indicates that the photo-signal may regulate NF-Y levels at early time points through the miR pathway.

The involvement of miR169 in photo-signaling has been indicated by earlier studies as well. It was shown that five members of the osa-miR169 family were up-regulated in phyB mutant, suggesting their regulations by the phytochrome [19]. The miR169 family was also regulated by exposure to UV-B radiation in Arabidopsis thaliana, Populus tremula and Triticum aestivum [38] [39]. Several reports also have indicated the role of NF-Y family, in light-mediated gene regulation. In Arabidopsis, NF-YA5 regulates the chlorophyll a/b binding protein 


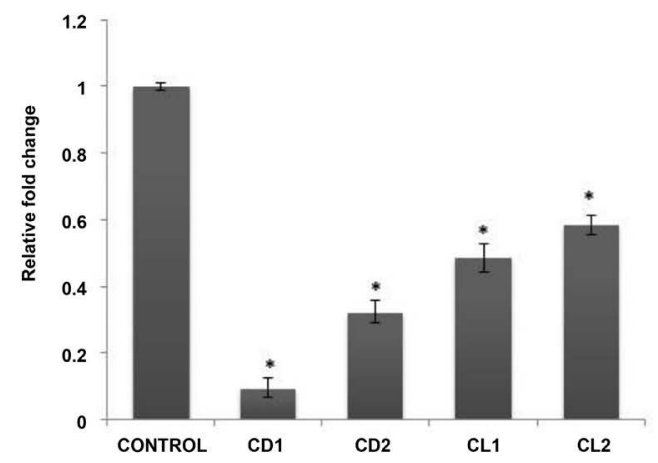

(a)

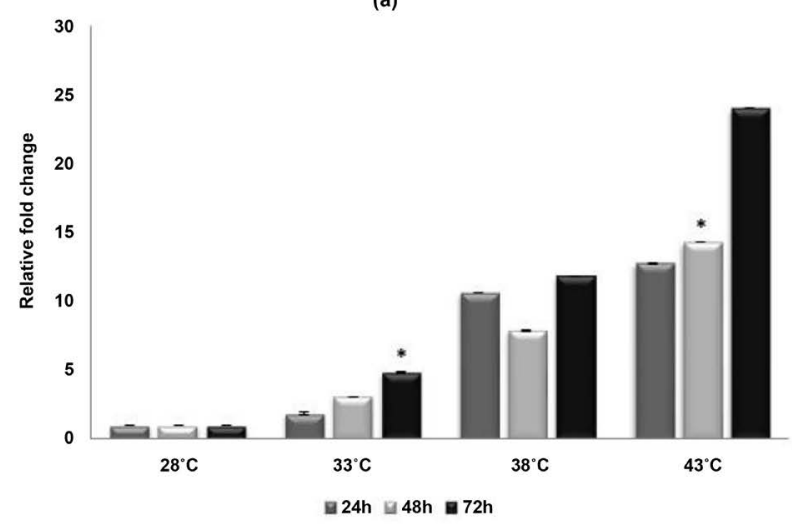

(c)

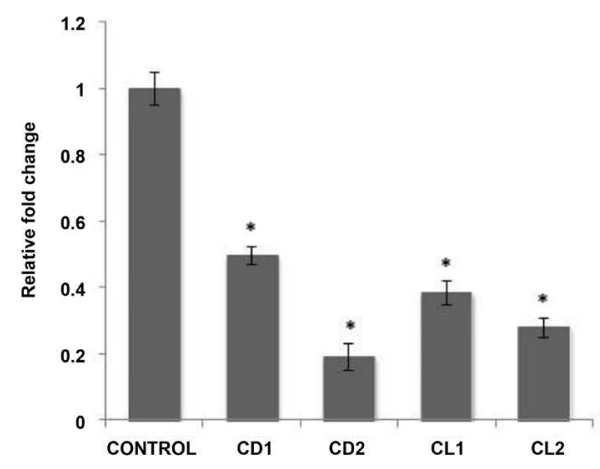

(b)

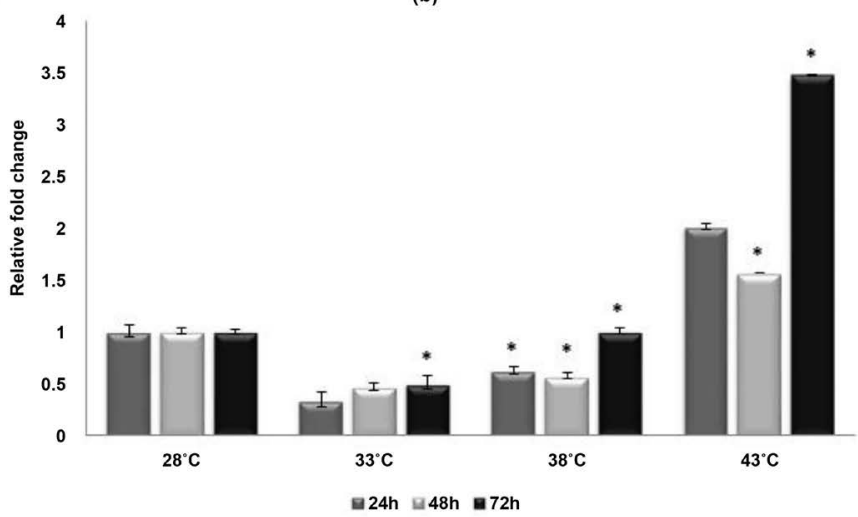

(d)

Figure 1. The relative fold change expression in leaves of seedlings exposed to continuous light (CL1 = 24 h, CL2 = $48 \mathrm{~h})$ and continuous darkness $(\mathrm{CD} 1=24 \mathrm{~h}, \mathrm{CD} 2=48 \mathrm{~h}$ ) of (a) osa-miR169 (b) nuclear transcription factor Y (LOC_Os07g06470). The expression was also analyzed in leaves of seedlings exposed to $33^{\circ} \mathrm{C}, 38^{\circ} \mathrm{C}$ and $43^{\circ} \mathrm{C}$ temperature for $24 \mathrm{~h}, 48 \mathrm{~h}$ and $72 \mathrm{~h}$ duration for (c) osa-miR169 (d) nuclear transcription factor Y (LOC_Os07g06470). The normalized expression values were calculated with respect to $18 \mathrm{~S}$ and fold change in expression was plotted. Control (c) was tissues obtained from unstressed plant grown at $28^{\circ} \mathrm{C} \pm 2{ }^{\circ} \mathrm{C}$ and $14 / 10 \mathrm{~h}$ photoperiod. Expression levels in each case were detected by qRT-PCR $(\mathrm{n}=3$; means \pm SDS). Asterisks indicate a significant difference of test conditions from their respective control samples $\left({ }^{*} \mathrm{p}<0.05\right.$; one-way ANOVA test).

expression in response to blue light [31]. A study conducted on Spinacia oleracea suggested that the binding of NF-Y complex at the promoter of the downstream signaling genes is controlled by the photo-signal [31]. The RNAi based knocking down of NF-YB2 in rice leads to reduced chlorophyll content in leaves and chloroplast degeneration. The constitutive over-expression of Ta-NF-YB3 in transgenic wheat lines also suggested its role in photosynthesis and early plant growth [40].

\subsection{High Temperature Effects the Expression of Osa-miR169 and Its Targets NF-Y}

To follow the effect of $\mathrm{HS}$ on osa-miR169 expression, $\mathrm{PB} 1$ seedlings were exposed to temperatures that were $5^{\circ} \mathrm{C}\left(33^{\circ} \mathrm{C}\right), 10^{\circ} \mathrm{C}\left(38^{\circ} \mathrm{C}\right)$ and $15^{\circ} \mathrm{C}\left(43^{\circ} \mathrm{C}\right)$ degree above normal. At each temperature the exposure time ranged to $24 \mathrm{~h}, 48 \mathrm{~h}$ and $72 \mathrm{~h}$. The data for expression profiles of osa-miR169 is provided in Figure 1(c). It was observed that the expression increased with increasing HS; with $43^{\circ} \mathrm{C}, 72 \mathrm{~h}$ having $>20$ folds higher relative expression in comparison to control. This indicates that osa-miR169 expression in PB1 is induced by increasing 
temperatures.

The dynamically regulated target, NF-Y showed a decreased expression at $33^{\circ} \mathrm{C}$ and $38^{\circ} \mathrm{C}$ of HS when compared with the control (Figure $1(\mathrm{~d})$ ), but increase in target expression was seen at $43^{\circ} \mathrm{C}, 72 \mathrm{~h}$ ( 2-folds). The anti-correlation of miR169: $\mathrm{NF}-\mathrm{Y}$ node at $43^{\circ} \mathrm{C}$ indicates that target expression may be under miR regulation at early time points of moderate stress. At higher intensities of HS, other factors may come into play thereby masking the effect of miR regulation. Moreover, NF-Y and osa-miR169 being large families may show varied expression with time in a tissue-specific manner. So, single-cell gene expression analysis will be helpful to fully appreciate the influence of changing target expression with respect to the various miR family member expressions.

\subsection{High Temperature Priming under Light Modulates the Expression of Osa-miR169 and Its Target NF-Y}

Priming or a short pre-exposure to stress is emerging as a natural mechanism to enhance the tolerance of crop plants to HS [3] [8]. The phenomenon is based on the plants' ability to retain stress memory that relates to better adaptability. There has been growing interest to unravel the interactive loops between light and temperature which most recently has been extended to establishing the role of light as an essential signal in the process of a strongly acquired thermo-tolerance response in potato [41].

We investigated the expression profiles of osa-miR169 in flag leaves (FL) of plants primed with $\mathrm{HS}$ before anthesis $(\mathrm{PBH})$ and after anthesis $(\mathrm{PAH})$ during the light phase. The expression patterns were compared to those obtained in plants subjected to direct HS before anthesis $(\mathrm{NBH})$ and after anthesis (NAH). The results showed differential expression of osa-miR169 under primed and non-primed conditions relative to the control. It was found that the expression level of osa-miR169 in PAH plants was down-regulated as compared to NAH. In primed plants the expression of osa-miR169 was more in plants at PAH as compared to $\mathrm{PBH}$, whereas in non-primed plants the expression of osa-miR169 was highly up-regulated at NAH as compared to NBH (Figure 2(a)). The expression of NF-Y was also checked and compared. An inverse relationship between miR169 \& NF-Y was observed under both priming and non-priming conditions. It was found that NF-Y expression level was highly up-regulated in $\mathrm{PBH}$ ( 1.2-folds) as compared to PAH, but the expression of NF-Y in non-primed plants was up-regulated in $\mathrm{NBH}(\sim 0.4$-folds) as compared to the NAH conditions (Figure 2(b)).

The results indicate that the target levels would be complementary to the miR levels and influence the plant development by regulating the respective targets. Thus PAH lowers the levels of NF-Y, while PBH increases its levels, indicating that $\mathrm{PBH}$ may protect the plants against HS. Reports on Petunia and Antirrhinum suggested that miR169 can be substituted for the function of APETALA 2 (AP2) to negatively regulate a family of NF-Y subunit A to modulate the downstream pathways that control organ identity, flowering time and fate of floral 


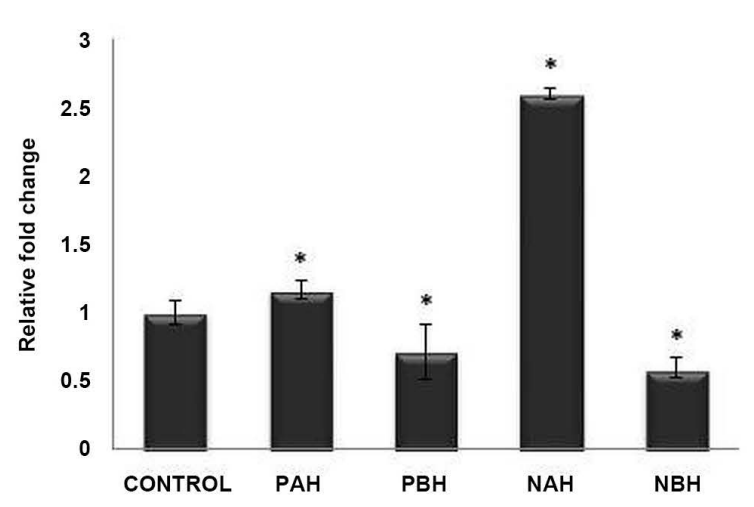

(a)

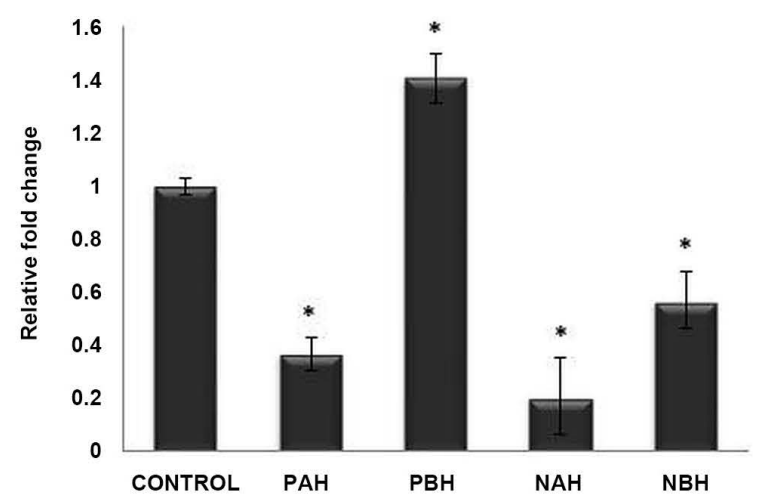

(b)

Figure 2. The relative fold change expression of (a) osa-miR169 (b) nuclear transcription factor Y (LOC_Os07g06470) in flag leaves of plants under control, priming ( $\mathrm{PAH}=$ Primed after anthesis, $\mathrm{PBH}=$ Primed before anthesis) and non-priming conditions $(\mathrm{NAH}=$ non-primed after anthesis, $\mathrm{NBH}=$ non-primed before anthesis). The normalized expression values were calculated with respect to $18 \mathrm{~S}$ and fold change in expression was plotted. Control (c) was tissues obtained from unstressed plant grown at $28^{\circ} \mathrm{C} \pm 2{ }^{\circ} \mathrm{C}$ and $14 / 10 \mathrm{~h}$ photoperiod. Expression levels in each case were detected by qRT-PCR $(\mathrm{n}=3$; means \pm SDS $)$. Significant differences of test conditions from their respective control samples are denoted by asterisks $\left({ }^{\star} \mathrm{P}<0.05\right.$; one-way ANOVA).

meristem [42]. The obtained results indicate that the expression of NF-Y and miR169 showed complementary regulation and this is supported by previous reports where it is shown that cross-talk mechanisms exist between light and temperature signalling [43]. In Arabidopsis, it has been demonstrated that NF-Y complex controls flowering time epigenetically by integrating environmental and developmental signals. NF-Y interacts with CONSTANS in the photoperiod pathway and DELLAs in the gibberellin pathway. Both pathways converge in the regulating the transcription of SOC1, a major floral pathway integrator as the NF-Y complex binds to a CCAAT box within the SOC1 promoter [44]. Thus NF-Y complexes act as critical mediators that regulate the response to environmental or intrinsic signals in plants [44].

\section{Conclusions}

In this study, we showed the involvement of osa-miR169 and target NF-Y module in adaptation to HS priming. The levels of osa-miR169 are highly up-regulated in HS non-primed plants as compared to primed plants in PB1 rice. The changes in miR levels indicate modulation of the corresponding transcript for NF-Y, which in turn may influence the interaction of NF-Y complex with other TFs. Thus miR169 levels will influence the expression of a number of downstream targets to regulate plant response under HS.

During the experiments, we observed that the grain yields, calculated in terms of grains per panicles, of HS primed (PBH and $\mathrm{PAH}$ ) rice plants were significantly higher than grain yields of non-primed (NBH and NAH) plants (data not shown). There cannot be a direct correlation of the two observations and the reasons for higher grain yields and require detailed investigation.

It is likely that the HS priming triggers the stress memory by influencing the miR169 expression levels which may then serve to enhance the HS tolerance re- 
sponse by modulating the levels of NF-Y. Since miR169 levels are also modulated by light, we hypothesize that the fine tuning of the whole pathway may be achieved by modulating the levels of miR169 by integrating the dual signals of light and temperature. The data obtained in this study will be useful to understand how plants acclimatize to overlapping cues from the changing environment. The present work also indicates the involvement of other miRs in the process so further study has been initiated to understand the molecular basis of light dependent HS priming. Other miR-target modules, which may play a role in HS memory are being further investigated through NGS sequencing and experimental validations. The detailed analysis of the regulation of miRs remains an important research question and it will be interesting to see how miRs integrate stress memory and light regulated development.

\section{Acknowledgements}

Authors are thankful for the funds received from International Centre for Genetic Engineering and Biotechnology, New Delhi and Science \& Engineering Research Board (SERB) Department of Science and Technology (DST), Government of India. SKS is thankful to SERB for Distinguished Fellowship Award. AKK is thankful to DST for research fellowship. AK is thankful to University Grants Commission, India, for research fellowship.

\section{Authors' Contributions Statement}

AKK and AK performed the experiments. NSM and SKS conceptualized and designed the experiments. All authors wrote, revised and approved the manuscript.

\section{Conflicts of Interest}

The authors declare no conflicts of interest regarding the publication of this paper.

\section{References}

[1] Lesk, C., Rowhani, P. and Ramankutty, N. (2016) Influence of Extreme Weather Disasters on Global Crop Production. Nature, 529, 84-87. https://doi.org/10.1038/nature16467

[2] Lorenzo, C.D., Sanchez-Lamas, M., Antonietti, M.S. and Cerdán, P.D. (2016) Emerging Hubs in Plant Light and Temperature Signaling. Photochemistry and Photobiology, 92, 3-13. https://doi.org/10.1111/php.12535

[3] Wang, X., Xin, C., Cai, J., Zhou, Q., Dai, T., Cao, W. and Jiang, D. (2016) Heat Priming Induces Trans-Generational Tolerance to High Temperature Stress in Wheat. Frontiers in Plant Science, 7, 501. https://doi.org/10.3389/fpls.2016.00501

[4] Chen, S. and Li, H. (2017) Heat Stress Regulates the Expression of Genes at Transcriptional and Post-Transcriptional Levels, Revealed by RNA-seq in Brachypodium distachyon. Frontiers in Plant Science, 7, 2067. https://doi.org/10.3389/fpls.2016.02067

[5] Hasanuzzaman, M., Nahar, K., Alam, M.M., Roychowdhury, R. and Fujita, M. (2013) 
Physiological, Biochemical, and Molecular Mechanisms of Heat Stress Tolerance in Plants. International Journal of Molecular Sciences, 14, 9643-9684. https://doi.org/10.3390/ijms14059643

[6] Iqbal, M., Raja, N., Yasmeen, F., Hussain, M., Ejaz, M. and Shah, M. (2017) Impacts of Heat Stress on Wheat: A Critical Review. Advances in Crop Science and Technology, 5, 1-9. https://doi.org/10.4172/2329-8863.1000251

[7] Ozga, J.A., Kaur, H., Savada, R.P. and Reinecke, D.M. (2016) Hormonal Regulation of Reproductive Growth under Normal and Heat-Stress Conditions in Legume and Other Model Crop Species. Journal of Experimental Botany, 68, 1885-1894. https://doi.org/10.1093/jxb/erw464

[8] Ling, Y., Serrano, N., Gao, G., Atia, M., Mokhtar, M., Woo, Y.H., Bazin, J., Veluchamy, A., Benhamed, M. and Crespi, M. (2018) Thermopriming Triggers Splicing Memory in Arabidopsis. Journal of Experimental Botany, 69, 2659-2675. https://doi.org/10.1093/jxb/ery062

[9] Richter, K., Haslbeck, M. and Buchner, J. (2010) The Heat Shock Response: Life on the Verge of Death. Molecular Cell, 40, 253-266.

https://doi.org/10.1016/j.molcel.2010.10.006

[10] Haslbeck, M. and Vierling, E. (2015) A First Line of Stress Defense: Small Heat Shock Proteins and Their Function in Protein Homeostasis. Journal of Molecular Biology, 427, 1537-1548. https://doi.org/10.1016/j.jmb.2015.02.002

[11] Han, S.-H., Park, Y.-J. and Park, C.-M. (2018) Light Primes the Thermally Induces Detoxification of Reactive Oxygen Species during Thermotolerance Development in Arabidopsis. Plant and Cell Physiology, 60, 230-241.

https://doi.org/10.1093/pcp/pcy206

[12] Jones-Rhoades, M.W., Bartel, D.P. and Bartel, B. (2006) MicroRNAs and Their Regulatory Roles in Plants. Annual Review of Plant Biology, 57, 19-53. https://doi.org/10.1146/annurev.arplant.57.032905.105218

[13] Barciszewska-Pacak, M., Milanowska, K., Knop, K., Bielewicz, D., Nuc, P., Plewka, P., Pacak, A.M., Vazquez, F., Karlowski, W. and Jarmolowski, A. (2015) Arabidopsis microRNA Expression Regulation in a Wide Range of Abiotic Stress Responses. Frontiers in Plant Science, 6, 410. https://doi.org/10.3389/fpls.2015.00410

[14] Stief, A., Brzezinka, K., Lämke, J. and Bäurle, I. (2014) Epigenetic Responses to Heat Stress at Different Time Scales and the Involvement of Small RNAs. Plant Signaling and Behavior, 9, e970430. https://doi.org/10.4161/15592316.2014.970430

[15] Sailaja, B., Voleti, S., Subrahmanyam, D., Sarla, N., Prasanth, V.V., Bhadana, V. and Mangrauthia, S. (2014) Prediction and Expression Analysis of miRNAs Associated with Heat Stress in Oryza sativa. Rice Science, 21, 3-12. https://doi.org/10.1016/S1672-6308(13)60164-X

[16] Ragupathy, R., Ravichandran, S., Mahdi, M.S.R., Huang, D., Reimer, E., Domaratzki, M. and Cloutier, S. (2016) Deep Sequencing of Wheat sRNA Transcriptome Reveals Distinct Temporal Expression Pattern of miRNAs in Response to Heat, Light and UV. Scientific Reports, 6, Article No. 39373. https://doi.org/10.1038/srep39373

[17] Hivrale, V., Zheng, Y., Puli, C.O.R., Jagadeeswaran, G., Gowdu, K., Kakani, V.G., Barakat, A. and Sunkar, R. (2016) Characterization of Drought- and Heat-Responsive microRNAs in Switchgrass. Plant Science, 242, 214-223.

https://doi.org/10.1016/j.plantsci.2015.07.018

[18] Chung, P.J., Park, B., Wang, H., Liu, J., Jang, I.-C. and Chua, N.-H. (2016) Light-Inducible miR163 Targets PXMT1 Transcripts to Promote Seed Germination and Primary Root Elongation in Arabidopsis. Plant Physiology, 170, 1772-1782. 
https://doi.org/10.1104/pp.15.01188

[19] Sun, W., Xu, X.H., Wu, X., Wang, Y., Lu, X., Sun, H. and Xie, X. (2015) Genome-Wide Identification of microRNAs and Their Targets in Wild Type and phyB Mutant Provides a Key Link between microRNAs and the phyB-Mediated Light Signaling Pathway in Rice. Frontiers in Plant Science, 6, 372. https://doi.org/10.3389/fpls.2015.00372

[20] Combier, J.-P., Frugier, F., De Billy, F., Boualem, A., El-Yahyaoui, F., Moreau, S., Vernié, T., Ott, T., Gamas, P. and Crespi, M. (2006) MtHAP2-1 Is a Key Transcriptional Regulator of Symbiotic Nodule Development Regulated by microRNA169 in Medicago truncatula. Genes and Development, 20, 3084-3088. https://doi.org/10.1101/gad.402806

[21] Hsieh, L.-C., Lin, S.-I., Shih, A.C.-C., Chen, J.-W., Lin, W.-Y., Tseng, C.-Y., Li, W.-H. and Chiou, T.-J. (2009) Uncovering Small RNA-Mediated Responses to Phosphate Deficiency in Arabidopsis by Deep Sequencing. Plant Physiology, 151, 2120-2132. https://doi.org/10.1104/pp.109.147280

[22] Gu, M., Xu, K., Chen, A., Zhu, Y., Tang, G. and Xu, G. (2010) Expression Analysis Suggests Potential Roles of microRNAs for Phosphate and Arbuscular Mycorrhizal Signaling in Solanum lycopersicum. Physiologia Plantarum, 138, 226-237. https://doi.org/10.1111/j.1399-3054.2009.01320.x

[23] Thiebaut, F., Grativol, C., Tanurdzic, M., Carnavale-Bottino, M., Vieira, T., Motta, M.R., Rojas, C., Vincentini, R., Chabregas, S.M. and Hemerly, A.S. (2014) Differential sRNA Regulation in Leaves and Roots of Sugarcane under Water Depletion. PLoS ONE, 9, e93822. https://doi.org/10.1371/journal.pone.0093822

[24] Li, Y., Fu, Y., Ji, L., Wu, C. and Zheng, C. (2010) Characterization and Expression Analysis of the Arabidopsis mir169 Family. Plant Science, 178, 271-280. https://doi.org/10.1016/j.plantsci.2010.01.007

[25] Cao, S., Kumimoto, R.W., Gnesutta, N., Calogero, A.M., Mantovani, R. and Holt, B.F. (2014) A Distal CCAAT/NUCLEAR FACTOR Y Complex Promotes Chromatin Looping at the FLOWERING LOCUS T Promoter and Regulates the Timing of Flowering in Arabidopsis. The Plant Cell, 26, 1009-1017. https://doi.org/10.1105/tpc.113.120352

[26] Leyva-González, M.A., Ibarra-Laclette, E., Cruz-Ramírez, A. and Herrera-Estrella, L. (2012) Functional and Transcriptome Analysis Reveals an Acclimatization Strategy for Abiotic Stress Tolerance Mediated by Arabidopsis NF-YA Family Members. PLoS ONE, 7, e48138. https://doi.org/10.1371/journal.pone.0048138

[27] Ni, Z., Hu, Z., Jiang, Q. and Zhang, H. (2013) GmNFYA3, a Target Gene of miR169, Is a Positive Regulator of Plant Tolerance to Drought Stress. Plant Molecular Biolo$g y$, 82, 113-129. https://doi.org/10.1007/s11103-013-0040-5

[28] Shi, Y., Ding Y. and Yang, S. (2014) Cold Signal Transduction and Its Interplay with Phytohormones during Cold Acclimation. Plant Cell Physiology, 56, 7-15. https://doi.org/10.1093/pcp/pcu115

[29] Li, C., Distelfeld, A., Comis, A. and Dubcovsky, J. (2011) Wheat Flowering Repressor VRN2 and Promoter $\mathrm{CO}_{2}$ Compete for Interactions with NUCLEAR FACOTR Y-Complexes. The Plant Journal, 67, 763-773. https://doi.org/10.1111/j.1365-313X.2011.04630.x

[30] Yamamoto, A., Kagaya, Y., Toyoshima, R., Kagaya, M., Takeda, S. and Hattori, T. (2009) Arabidopsis NF-YB Subunits LEC1 and LEC1-LIKE Activate Transcription by Interacting with Seed-Specific ABRE-Binding Factors. The Plant Journal, 58, 843-856. https://doi.org/10.1111/j.1365-313X.2009.03817.x 
[31] Petroni, K., Kumimoto, R.W., Gnesutta, N., Calvenzani, V., Fornari, M., Tonelli, C., Holt, B.F. and Mantovani, R. (2012) The Promiscuous Life of Plant NUCLEAR FACTOR Y Transcription Factors. The Plant Cell, 24, 4777-4792. https://doi.org/10.1105/tpc.112.105734

[32] Khan, A., Goswami, K., Sopory, S.K. and Sanan-Mishra, N. (2017) "Mirador" on the Potential Role of miRNAs in Synergy of Light and Heat Networks. Indian Journal of Plant Physiology, 22, 587-607. https://doi.org/10.1007/s40502-017-0329-5

[33] Mittal, D., Mukherjee, S.K., Vasudevan, M. and Mishra, N.S. (2013) Identification of Tissue-Preferential Expression Patterns of Rice miRNAs. Journal of Cellular Biochemistry, 114, 2071-2081. https://doi.org/10.1002/jcb.24552

[34] Devers, E.A., Branscheid, A., May, P. and Krajinski, F. (2011) Stars and Symbiosis: microRNA- and microRNA*-Mediated Transcript Cleavage Involved in Arbuscular Mycorrhizal Symbiosis. Plant Physiology, 156, 1990-2010. https://doi.org/10.1104/pp.111.172627

[35] Sorin, C., Declerck, M., Christ, A., Blein, T., Ma, L., Lelandais-Brière, C., Njo, M.F., Beeckman, T., Crespi, M. and Hartmann, C. (2014) A miR169 Isoform Regulates Specific NF-YA Targets and Root Architecture in Arabidopsis. New Phytologist, 202, 1197-1211. https://doi.org/10.1111/nph.12735

[36] Zhao, B., Ge, L., Liang, R., Li, W., Ruan, K., Lin, H. and Jin, Y. (2009) Members of miR-169 Family Are Induced by High Salinity and Transiently Inhibit the NF-YA Transcription Factor. BMC Molecular Biology, 10, 29. https://doi.org/10.1186/1471-2199-10-29

[37] Sato, H., Mizoi, J., Tanaka, H., Maruyama, K., Qin, F., Osakabe, Y., Morimoto, K., Ohori, T., Kusakabe, K. and Nagata, M. (2014) Arabidopsis DPB3-1, a DREB2A Interactor, Specifically Enhances Heat Stress-Induced Gene Expression by Forming a Heat Stress-Specific Transcriptional Complex with NF-Y Subunits. The Plant Cell, 26, 4954-4973. https://doi.org/10.1105/tpc.114.132928

[38] Zhou, X., Wang, G. and Zhang, W. (2007) UV-B Responsive microRNA Genes in Arabidopsis thaliana. Molecular Systems Biology, 3, 103. https://doi.org/10.1038/msb4100143

[39] Jia, X., Ren, L., Chen, Q.-J., Li, R. and Tang, G. (2009) UV-B-Responsive Micrornas in Populus tremula. Journal of Plant Physiology, 166, 2046-2057. https://doi.org/10.1016/j.jplph.2009.06.011

[40] Stephenson, T.J., Mcintyre, C.L., Collet, C. and Xue, G.-P. (2011) TaNF-YB3 Is Involved in the Regulation of Photosynthesis Genes in Triticum aestivum. Functional and Integrative Genomics, 11, 327-340. https://doi.org/10.1007/s10142-011-0212-9

[41] Trapero-Mozos, A., Ducreux, L.J., Bita, C.E., Morris, W., Wiese, C., Morris, J.A., Paterson, C., Hedley, P.E., Hancock, R.D. and Taylor, M. (2018) A Reversible Lightand Genotype-Dependent Acquired Thermotolerance Response Protects the Potato Plant from Damage Due to Excessive Temperature. Planta, 247, 1377-1392. https://doi.org/10.1007/s00425-018-2874-1

[42] Li, S., Castillo-González, C., Yu, B. and Zhang, X. (2017) The Functions of Plant Small RNAs in Development and in Stress Responses. The Plant Journal, 90, 654-670. https://doi.org/10.1111/tpj.13444

[43] Serivichyaswat, P.T., Susila, H. and Ahn, J.H. (2017) Elongated Hypocotyl 5 Homo$\log (\mathrm{HYH})$ Negatively Regulates Expression of the Ambient Temperature-Responsive mictoRNA Gene MIR169. Frontiers in Plant Science, 8, 2087. https://doi.org/10.3389/fpls.2017.02087

[44] Hou, X., Zhou, J., Liu, C., Liu, L., Shen, L. and Yu, H. (2014) Nuclear Factor Y-Mediated 
H3K27me3 Demethylation of the SOC1 Locus Orchestrates Flowering Responses of Arabidopsis. Nature Communications, 5, Article No. 4601.

https://doi.org/10.1038/ncomms5601

\section{Appendix}

Table A1. List of primers.

\begin{tabular}{ccc}
\hline Gene & Primer & Sequence (5'-3') \\
\hline miR169 (FP) & Forward & CCACGACTAGCCAAGGATGAC \\
miR169 (SL) & Stem-loop & GTCGTATCCAGTGCAGGGTCCGAGGTATTCGCACTGGAT \\
URP (SL) & Reverse & ACGACCCGGCA \\
18S_Fwd & Forward & CCAGTGCAGGGTCCGAGGTA \\
18S_Rev & Reverse & CTACGTCCCTGCCCTTTGTACA \\
NF-Y_Fwd & Forward & ACACTTCACCGGACCATTCAA \\
NF-Y_Rev & Reverse & GCACACACACACACTGCATCAG \\
\hline
\end{tabular}

